\title{
Study of Total Ionizing Dose Induced Read Bit Errors in Magneto-resistive Random Access Memory
}

Zhang Haohao ${ }^{1223)}$, Bi Jinshun ${ }^{12) 33 \dagger}$, Wang Haibin ${ }^{4)}$, Hu Hongyang ${ }^{1223)}$, Li Jin ${ }^{1223)}$, Ji Lanlong ${ }^{1223)}$, Liu Ming ${ }^{12) 33}$

1) (The Key Laboratory of Microelectronics Devices \& Integrated Technology, Institute of Microelectronics of Chinese Academy of Sciences, Beijing 100029, China)

2) (Jiangsu Synergetic Innovation Center for Advanced Materials(SICAM), Nanjing 210009, China)

3) (The Key Laboratory of Microelectronics Devices \& Integrated Technology, Institute of Microelectronics of Chinese Academy of Sciences, Beijing 100029, China)

4) (School of Internet of Things Engineering, HoHai University, Changzhou, China)

\begin{abstract}
This paper presents the Co-60 irradiation results for a $16 \mathrm{Mb}$ Magneto-resistive Random Access Memory (MRAM). Read bit errors were observed during Total Ionizing Dose (TID) testing. We have investigated their physical mechanisms and proposed a resistance drift model of the access transistor in 1M1T (a magnetic tunnel junction and a transistor) storage structure to understand the phenomenon. Read operations have been simulated by HSPICE simulator with the magnetic tunnel junction (MTJ) compact model. The simulation results reveal that the resistance shift of access transistors has a great impact on read bit errors in MRAM. The experimental data and analysis in this work can be used to harden MRAM designs targeting space-borne applications.
\end{abstract}

Key Words: MRAM, total ionizing dose effects, read bit errors, SPICE model, radiation-harden.

†corresponding author. E-mail: bijinshun@ime.ac.cn 


\section{Introduction}

In the space radiation environment, there are a variety of extremely energetic particles, such as protons, electrons, and heavy ions ${ }^{[1-2]}$. Ion strikes may alter the insulator's properties in metal-oxide-semiconductor field-effect transistor (MOSFET) and consequently induce radiation effects. These effects have been identified as the major reliability issues for microelectronic systems. As total ionizing dose effects pose a long-term impact on electronic devices, it is not surprising that these effects and their mitigation techniques for devices targeting harsh radiation environments ${ }^{[3]}$ have been studied by the community.

A majority of modern system on chips (SOCs) are constructed with memories and they may occupy up to $95 \%$ of the overall die area ${ }^{[4]}$. With wide applications of SOCs in spacecrafts and satellites, space-grade electronic systems call for novel non-volatile memory devices featuring both higher density and reliability ${ }^{[5-7]}$. Magneto-resistive Random Access Memory is believed to be one of the promising candidates. This is because it has unique advantage compared to conventional memories (e.g., SRAM and FLASH) in terms of speed, integration density, unlimited endurance, less power consumption ${ }^{[8-10]}$, and inherent immunity to radiation strikes ${ }^{[11-13]}$. Each MRAM bit-cell is composed of an access transistor and a MTJ (Magnetic Tunnel Junction). The MTJ device has a free ferromagnetic layer and a pinned ferromagnetic layer separated by a thin insulator layer. Due to the parallel or anti-parallel magnetizations of these two ferromagnetic layers, MTJs exhibit two stable resistance states, low or high ${ }^{[14]}$.

Previous TID testing results have demonstrated that neither the electrical nor the magnetic properties of MTJs exhibited errors induced by radiation strikes ${ }^{[12,13]}$. However, during our experiments, we observed read bit errors in MRAM chips after total dose reached a certain threshold value. To our best knowledge, there are no complete or reasonable explanations w.r.t. how TID affects MRAM storage cells and consequently leads to read bit errors.

In this work, we investigate TID effects on MRAM devices induced by Cobalt-60. Main electrical parameters of MRAM are monitored and read bit errors as a function of total dose are investigated. We also present a resistance drift model to understand the read bit error's phenomenon induced by TID radiation.

\section{DUT and Test Procedure}

The Device Under Test (DUT) in our work is a commercial MRAM device-MR4A08B, the vendor of which is Everspin Corporation. It has $16 \mathrm{Mb} 1 \mathrm{M} 1 \mathrm{~T}$ (1 MTJ and 1 access transistor) storage cells. The access transistor features gate oxide of $2.8-\mathrm{nm}$ thickness and $\mathrm{W} / \mathrm{L}=0.8 \mu \mathrm{m} / 0.18 \mu \mathrm{m}$. The shallow trench isolation (STI) is introduced for electrical isolation with the depth of approximately $380 \mathrm{~nm}$. The MRAM devices feature $3.3 \mathrm{~V}$ power supply, 8 bits wordwidth, $9 \mathrm{~mA}$ AC standby current, and SRAM-compatible $35 \mathrm{~ns}$ access time with unlimited endurance. MR4A08B utilizes 44-lead plastic TSOP package.

The TID radiation experiments were performed at the Department of Technical Physics of Peking University with a cobalt-60 radiation source at a dose rate of $50 \mathrm{rad}(\mathrm{Si}) / \mathrm{s}$. Electrical 
measurements were conducted at $0,20,30,40,45,50,55,65,75 \mathrm{krad}(\mathrm{Si})$ respectively. During TID irradiation, the DUTs were biased at the worst-case condition. The enable pins of MRAM (/E, /W, /G) were pulled up, address and data pins were connected to 3.3V. All the DUTs were initially programmed with the checkerboard pattern (i.e.,55H) and read back between each dose step. Subsequently, electrical parameters were measured. Finally, the pattern of all-0(all-1) was tested and March_6N test were performed. March_6N is a memory test approach designed to uncover a variety of faults. It is formally written as:

$\{\uparrow(\mathrm{w} 55 \mathrm{H}) ; \uparrow(\mathrm{r} 55 \mathrm{H}, \mathrm{wAAH}) ; \downarrow(\mathrm{rAAH}, \mathrm{w} 55 \mathrm{H}) ; \uparrow(\mathrm{r} 55 \mathrm{H})\}$

The sequences are:

write 55 with ascending order of address;

read 55 and write AA with ascending order;

read AA and write 55 with descending order;

finally read 55 with ascending order.

This test procedure was repeated at each irradiation step until total dose was accumulated up to $75 \mathrm{krad}(\mathrm{Si})$. This is in accordance with the specifications in MIL-STD-883D Test Method 1019.4 -- start electrical characterization within one hour after exposure and finish within two hours before the next run. All the parameters of the DUTs were measured with the Verigy 93000 SOC test system. After the final irradiation step, the DUTs were annealed at room temperature up to 120 hours with pins electrically floating.

\section{Experimental Results and Discussion}

\subsection{Read bit errors and DC parameters versus TID}

Below $55 \mathrm{krad}(\mathrm{Si})$ dose level, all the DUTs remained fully functional. After the dose was accumulated to $65 \mathrm{krad}(\mathrm{Si})$, all the devices started to read out erroneous data when the initial data pattern was $55 \mathrm{H}$. When read bit errors were detected, error types and their corresponding logic address were logged. Error distribution differs from each DUT and random logic address were observed. The read bit error counts and types are summarized in Table 1. ' $1 \rightarrow 0$ ' means the bit is initially 1 and then flips to 0 . In fact, DUTs 1 through 3 observed 1, 5169 and 30 bit errors, respectively.

When the accumulated total dose surpassed the threshold value, read error bits started to grow exponentially ${ }^{[14,15]}$. Due to process variation, these DUTs exhibited different TID radiation threshold values around $65 \mathrm{krad}(\mathrm{Si}) .65 \mathrm{krad}(\mathrm{Si})$ may be higher than the TID threshold of DUT2, and thus, DUT2 showed more bit errors compared with other DUTs.

Table 2 compiles the number of multiple upset per byte for each operation of reading ' $55 \mathrm{H}$ '. Fig. 1 shows the standby current $\mathrm{I}_{\mathrm{SB}}$ as a function of the irradiation dose and annealing time. As can be seen, $\mathrm{I}_{\mathrm{SB}}$ rises sharply starting $75 \mathrm{krad}(\mathrm{Si})$ and surpasses the specification limit of $9 \mathrm{~mA}$. The other DC parameters show the same variation trend as standby current. 


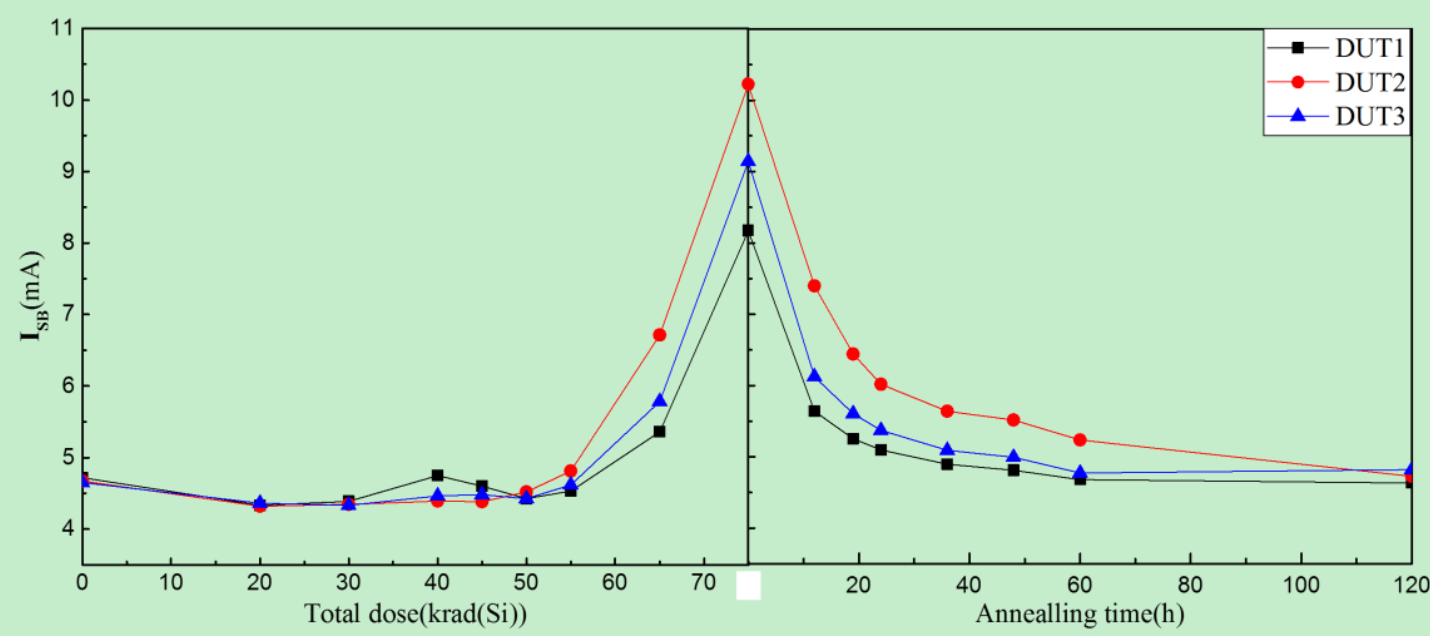

Fig.1. standby current as a function of total dose and annealing time

Table 1. Read error types and count caused by TID effects in reading initial ' $55 \mathrm{H}$ ' pattern.

\begin{tabular}{|c|c|c|c|c|}
\hline \multirow{2}{*}{ Samples } & \multirow{2}{*}{ Total dose } & \multirow{2}{*}{ Error count } & \multicolumn{2}{|c|}{ Error types } \\
\hline & & & $0 \rightarrow 1$ & $1 \rightarrow 0$ \\
\hline DUT1 & & 1 & 0 & 1 \\
\hline DUT2 & $65 \mathrm{krad}(\mathrm{Si})$ & 5169 & 1060 & 4109 \\
\hline DUT3 & & 30 & 11 & 19 \\
\hline DUT1 & & 3319 & 1233 & 2086 \\
\hline DUT2 & $75 \mathrm{krad}(\mathrm{Si})$ & 11864 & 1099 & 10765 \\
\hline DUT3 & & 9415 & 1059 & 8356 \\
\hline
\end{tabular}

Table 2. Multiple upset per byte caused by ionizing irradiation

\begin{tabular}{|c|c|c|c|c|c|c|}
\hline \multirow{2}{*}{ Sample } & \multirow{2}{*}{ Total dose } & \multirow{2}{*}{ Total bytes } & \multicolumn{4}{|c|}{ Multiple upset per byte } \\
\hline & & & 2 bits & 3 bits & 4 bits & 5 bits \\
\hline DUT1 & \multirow{3}{*}{$65 \mathrm{krad}(\mathrm{Si})$} & 0 & 0 & 0 & 0 & 0 \\
\hline DUT2 & & 112 & 110 & 2 & 0 & 0 \\
\hline DUT3 & & 6 & 2 & 1 & 2 & 1 \\
\hline DUT1 & \multirow{3}{*}{$75 \mathrm{krad}(\mathrm{Si})$} & 76 & 70 & 5 & 1 & 0 \\
\hline DUT2 & & 1764 & 1251 & 396 & 114 & 3 \\
\hline DUT3 & & 1022 & 917 & 104 & 1 & 0 \\
\hline
\end{tabular}

3.2 The resistance drift of access transistor during TID irradiation

Fig. 2 displays the cross-sectional TEM (Transmission Electron Microscope) view of the $1 \mathrm{M} 1 \mathrm{~T}$ storage cell. The memory is fabricated on a 180nm CMOS process with 5 metal layers 
and the MTJ is created during backend process steps between the last two metallization layers. As peripheral circuits have been demonstrated to be functional throughout our experiments, we have therefore studied TID effects on the access transistor in 1M1T storage cell.
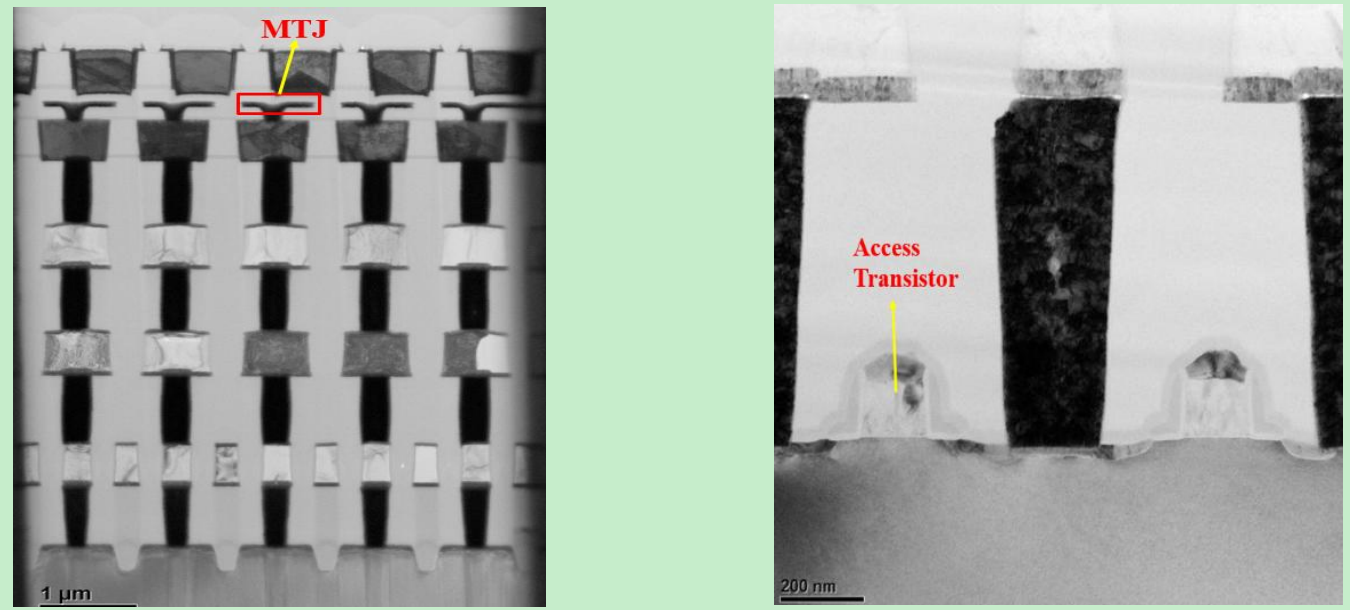

Fig.2. Cross-sectional TEM view of 1M1T memory cell

Even though the MTJ itself is insensitive to TID effects, the radiation source may still degrade the performance of access transistors. In the structure of 1M1T, the gate oxide thickness of the access transistor is $2.8 \mathrm{~nm}$ and is believed to be less sensitive to TID exposure. This is because reduction in gate oxide thickness essentially eliminates radiation-induced performance degradation, as discussed in previous results ${ }^{[16-18]}$. By contrast, the STI oxide thickness is $380 \mathrm{~nm}$ and does not scale down correspondingly, TID radiation will induce significant charge trapping in STI region ${ }^{[19,20]}$.

Prior to irradiation, the channel resistance of access transistor ${ }^{[21]}$ is calculated as

$$
R_{\text {mos }}=\frac{L}{W} \cdot \frac{1}{u_{n} C_{o x}\left(V_{g s}-V_{t}\right)}(1)
$$

where $L$ and $W$ represent the length and width of transistor respectively, $C_{o x}$ is gate oxide capacitance per unit area, $V_{g s}$ is the voltage between gate and source region, $V_{t}$ is the threshold voltage, $u_{n}$ is carrier mobility. The linear channel resistance is given in Function (1), as can be seen, small changes in carrier mobility or threshold voltage of access transistor would lead to the obvious variations of channel resistance.

Fig. 3 shows the structure of STI parasitic transistor where the trench oxide serves as the thick gate oxide. In a 1M1T storage cell, the access transistor is composed of a main transistor and two parasitic edge transistors. The threshold voltage shift of the STI parasitic transistor induced by TID effects is given by:

$$
\Delta V_{t}=\Delta V_{o t}+\Delta V_{i t}=-\frac{q\left(\Delta N_{o t}+\Delta N_{i t}\right) t_{O X}}{\varepsilon_{0} \varepsilon_{O X}}
$$

where $\Delta V_{o t}$ and $\Delta V_{i t}$ are the voltage shifts caused by oxide trapped charge and interface trapped charge respectively. $\Delta N_{o t}$ is the oxide trapped charge density, $\Delta N_{i t}$ means the interface trapped charge density, and $t_{O X}$ is the thickness of gate oxide. 

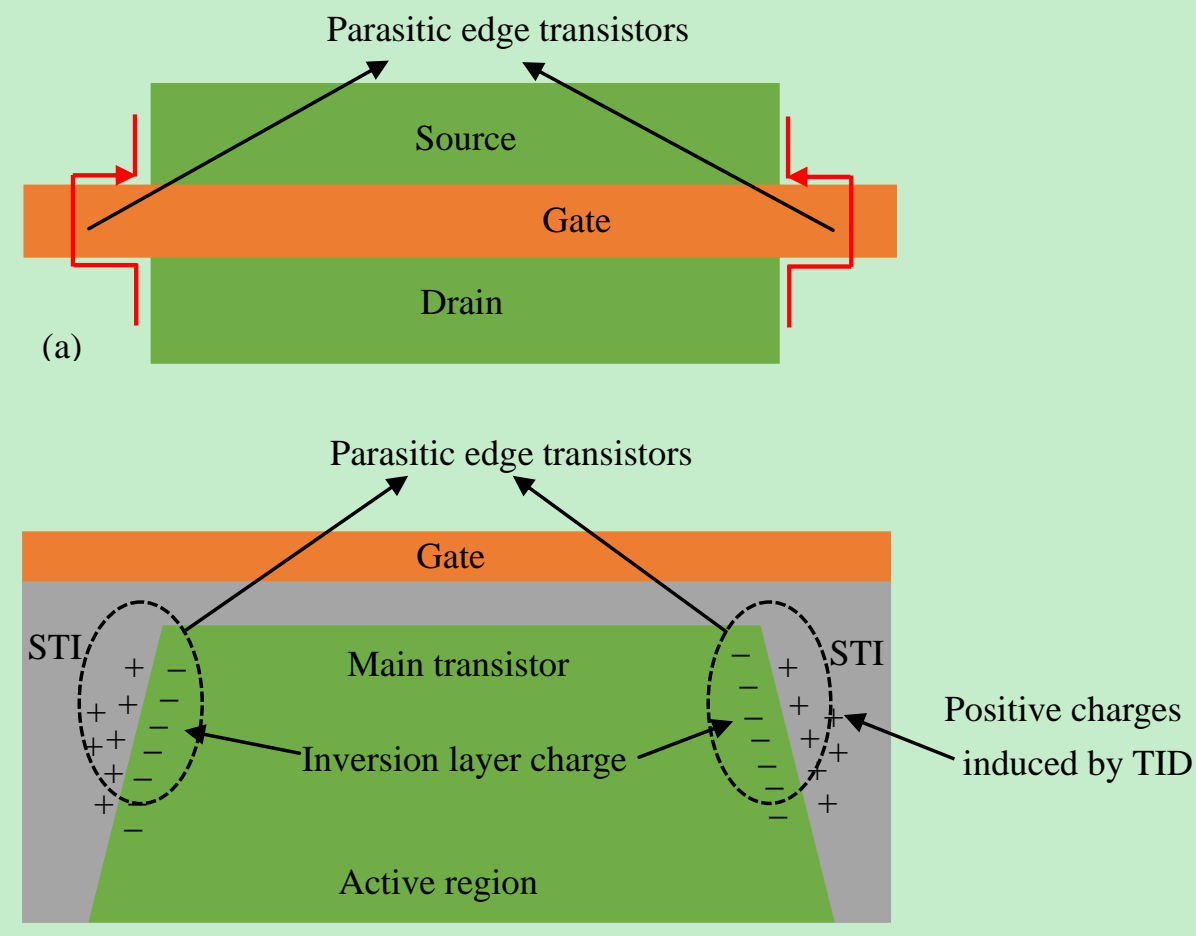

(b)

Fig. 3. (a)The top and (b) gate-length-direction cross-sectional views of parasitic edge transistor in NMOS after TID irradiation.

After TID irradiation, the channel resistance of access transistor $R_{m o s}^{\prime}$ becomes:

$$
R_{m o s}^{\prime}=\frac{L}{W+\Delta w} \frac{1+a_{i t} \Delta N_{i t}}{u_{0} C_{o x}} \frac{1}{\left[V_{g s}-V_{t}+\frac{q}{C_{o x}}\left(\Delta N_{o t}+\Delta N_{i t}\right)\right]}
$$

where $\mu_{0}$ is the effective mobility before irradiation, $\alpha_{i t}$ is a factor related to the interface trapped charge density.

For the NMOS device, as the accumulated total dose increases, more net positive charge would be trapped by the oxide and built up in the STI region. This will result in an increase of $\Delta w$ in the effective width of the main transistor ${ }^{[21]}$ as well as a larger negative shift of the threshold voltage of the parasitic transistors. After that, the channel resistance would show a decreasing change as illustrated in Function (3).

With the generation of interface charge, owing to its negative polarity ${ }^{[22]}$ and neutralization with parts of oxide trapped charge, a positive shift of the threshold voltage would be expected, albeit the slope of the shift is very slow. Because of the accumulation of interface trapped charge and carrier scattering effect, the carrier mobility $u_{n}$ would get degraded and the channel resistance of access transistor $R_{m o s}^{\prime}$ would increase. In our experiment, after annealing the DUTs for 120 hours at room temperature, all the DUTs recovered its normal functions. Interface charge appears to start annealing above about $100^{\circ} \mathrm{C}$ (most often at even higher temperatures) ${ }^{[23]}$, hence it indicates that very few interface charges can be generated. As a consequence, compared with oxide charge, the interface charge has an insignificant impact on the resistance drift.

As discussed above, the channel resistance of access transistor mainly shows a decreasing trend during TID irradiation. 

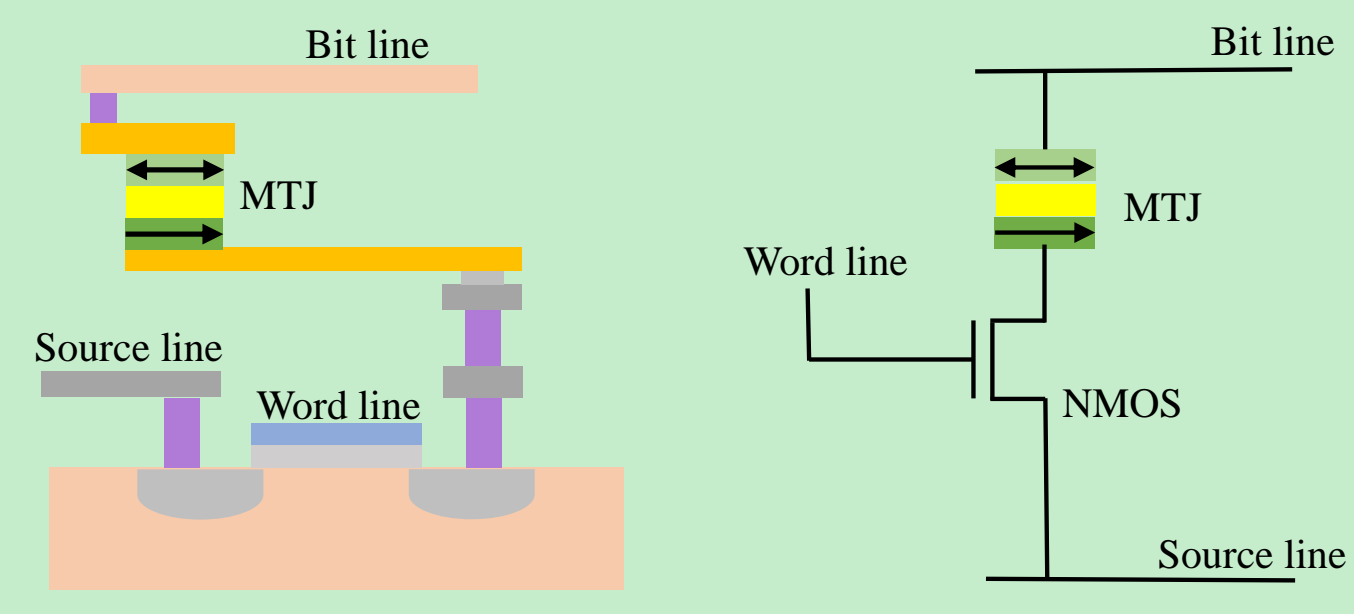

Fig.4. (a) The illustration of MRAM storage cell (b) the circuit schematic of 1M1T in MRAM

The structure of MRAM storage cell is illustrated in Fig.4. Assume that the high and low resistance of MTJ is $R_{H}$ and $R_{L}$ respectively, the reference cell's resistance is given by $R_{\text {ref }}=\left(R_{L}+R_{H}\right) / 2$. During a read operation, a read current $I_{\text {read }}$ is applied to both the data cell and reference cell simultaneously. The voltage drop across the target data cell is given by $V_{\text {sens }}=I_{\text {read }}\left(R_{m t j}+R_{m o s}\right)$, where $R_{m t j}$ represents the resistance of MTJ, $R_{H}$ or $R_{L}, R_{m o s}$ is the MOS channel resistance between source and drain region. The reference voltage is given by $V_{\text {ref }}=I_{\text {read }}\left(R_{\text {ref }}+R_{\text {mos }}\right)$. $V_{\text {sens }}$ and $V_{\text {ref }}$ would be compared by sense amplifier to determine the storage data -- ' 0 ' or ' 1 '. For example, if the MTJ is in high resistance state, the sensed voltage on $1 \mathrm{M} 1 \mathrm{~T}$ is higher than that on the reference bit cell and the output would be logic ' 1 '. The storage value can be distinguished by

$$
I_{\text {read }}\left(R_{L}+R_{\text {mos }}\right)<I_{\text {read }}\left(R_{\text {ref }}+R_{\text {mos }}\right)<I_{\text {read }}\left(R_{H}+R_{\text {mos }}\right)
$$

As indicated by the solid line in Fig. 5, the read sensed voltage has enough margin to distinguish high and low states under normal conditions (i.e., no irradiation).

Under TID irradiation, the induced parasitic transistor is connected with the main transistor, which could be viewed as a modulation effect of channel resistance. The resistance drift of $1 \mathrm{M} 1 \mathrm{~T}\left(R_{m t j}+R_{m o s}\right)$ would be expected, as discussed in section 3.2. This effect could eventually shift the sensed output voltage, and subsequently leads to a read bit error.

As illustrated by the dotted line in Fig. 5, if the negative voltage drift on data cell is larger than that on the reference cell, parts of high resistance state were mistaken for low resistance by comparing with the sensed reference voltage, leading to the read bit error of " $1 \rightarrow 0$ ". Besides, as the reading sensed margin becomes narrower than pre-irradiation, the memory becomes more susceptible to noise. The read failures occurs when the sensing circuit cannot distinguish between two states of the data cell. 


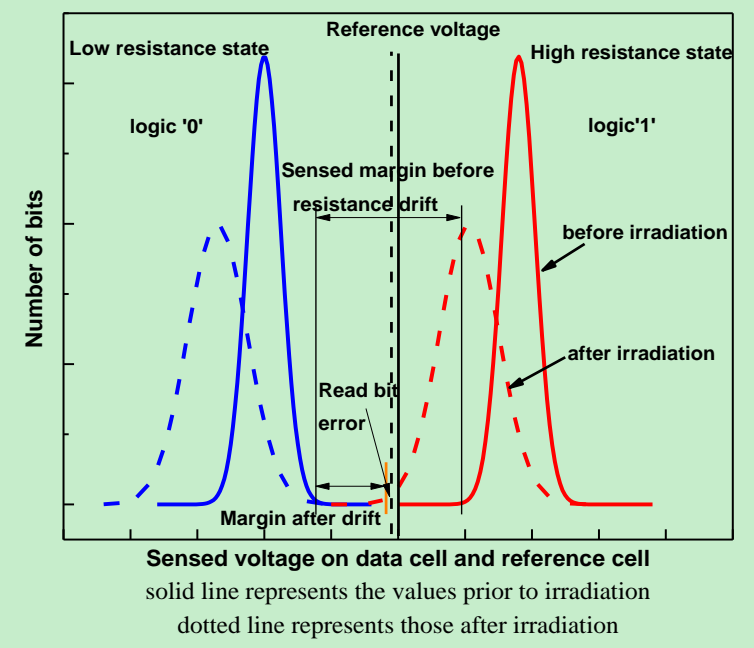

Fig. 5. Sensed voltage drift on the bit cell and read margin drift before and after irradiation (solid line refers to the values prior to irradiation, dotted line refers to those after irradiation)

\section{Resistance drift model evaluation}

In order to validate our hypothesis about the read bit errors induced by TID effects in the MRAM device, a conventional pre-charge sense amplifier ${ }^{[24]}$ is used for MRAM read operation, as shown in Fig.6. The sense circuit includes a reference branch for 1M1T reference cell and a data branch for data cell. Each branch is composed of a load PMOS, a clamp NMOS and a read enable NMOS. The clamp NMOS is used to ensure a low sense current when a proper voltage level is applied, and it is turned on to enable the read operation by setting RE as high. The access transistors in 1M1T share the same word line WL. A 1M1T storage cell is selected as the reference cell. During read operation, owing to the resistance difference between the reference cell path and data cell path, the current flowing through the data cell path will be different from that through the reference cell path. After the read path is turned on, because the load PMOS acts as a resistor, the current difference will be converted to a voltage difference between $V_{\text {ref }}$ and $V_{\text {data }}$. A sense amplifier detects the voltage difference and hence reads the stored data on node $\mathrm{Q}$.

In our simulation method, the resistance drift of access transistors is modeled by a parallel connection-a temporal transistor parallel with the access transistor. This is illustrated in Fig.6. The temporal transistor is used to simulate the parasitic edge transistor during TID irradiation. The parallel connection can lead to an increase in the effective width of access transistor as well as the resistance drift. Besides, it will result in the variation of sub-threshold leakage current of access transistor. This simulation method is consistent with the theoretical analysis above. Hence, the TID effects on access transistors could be simulated through the parallel connection.

It is well known that bias conditions significantly affect TID degradation on transistors ${ }^{[25]}$, the electrical shifts of access transistors M4 and M8 will be different after Co60 irradiation. In fact, the resistance of the reference cell is different from that of the data cell, and thus, the voltage levels at nodes 1 and 2 are different. In our simulations, these two parallel temporal transistors are set as different resistance values $\left(\mathrm{R}_{\mathrm{rad} 1}\right.$ and $\left.\mathrm{R}_{\mathrm{rad} 2}\right)$. 


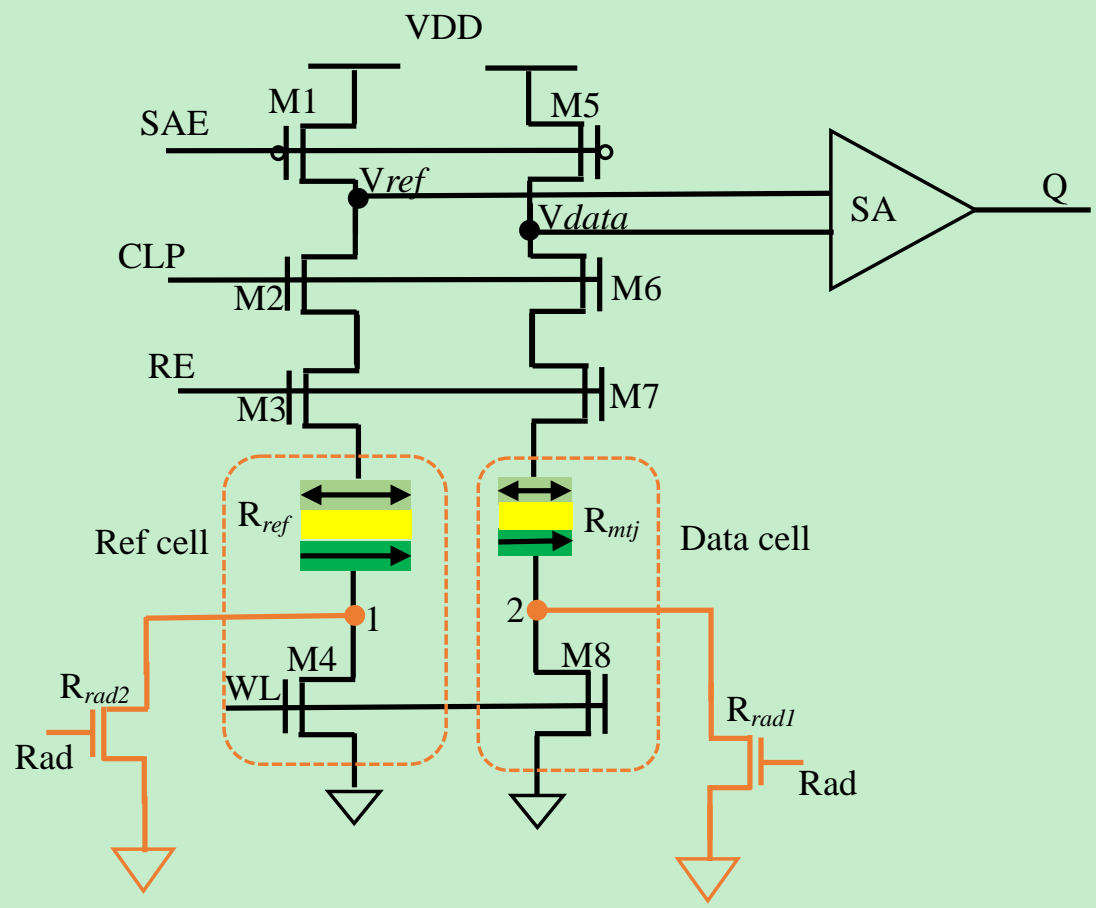

Fig.6. The simplified circuit scheme for 1M1T read operation

When the total dose surpasses the failure threshold value, the temporal transistors are switched on by the applied voltage Rad. Thus, the equivalent resistance of the parallel path formed by temporal transistor and access transistor becomes $R_{\text {mos }} \| R_{\text {rad }}$, which is actually $R_{m o s}^{\prime}$ in Equation (3) above. This simulates the TID-induced modulation effect on channel resistance.

The MTJ SPICE model from University of Minnesota ${ }^{[26]}$ and TSMC $0.18 \mu \mathrm{m}$ process model $^{[27]}$ are adopted for MRAM cell read operation simulations. The main parameters of MOSFET and MTJ were shown in Table 3.

Table 3. the parameters of MOSFET and MTJ in simulations

\begin{tabular}{lllll}
\hline MOSFET & W/L & MTJ & parameter & value \\
\hline M1/M5 & $4.5 \mu \mathrm{m} / 0.18 \mu \mathrm{m}$ & Free layer width & $\mathrm{w}$ & $70 \mathrm{~nm}$ \\
\hline M2/M6 & $1 \mu \mathrm{m} / 0.18 \mu \mathrm{m}$ & Free layer length & 1 & $70 \mathrm{~nm}$ \\
\hline M3/M7 & $1.8 \mu \mathrm{m} / 0.18 \mu \mathrm{m}$ & Free layer thickness & $\mathrm{t}$ & $1.498 \mathrm{~nm}$ \\
\hline M4/M8 & $0.8 \mu \mathrm{m} / 0.18 \mu \mathrm{m}$ & Saturation magnetization & $\mathrm{M}_{\mathrm{s} 0}$ & 1210 \\
\hline & & Polarization factor & $\mathrm{P}_{0}$ & 0.69 \\
\hline & & Resistance-area product & $\mathrm{RA}$ & $7 \Omega \cdot \mathrm{um}^{2}$ \\
\hline
\end{tabular}

The simulation results are presented in Figs.7-10. $V_{\text {data }}$ is equal to $V_{a p}$ and $V_{p}$ for high and low resistance states of the data cell respectively. Data cell is read twice starting from $2.1 \mathrm{~ns}$ and $15.1 \mathrm{~ns}$ respectively. The read bit errors occur when the total dose is accumulated to $65 \mathrm{krad}(\mathrm{Si})$, so the temporal transistors are turned on to simulate the impact of resistance drift on read operation at $15 \mathrm{~ns}$.

Fig.7 shows the read voltage margin between $V_{\text {ref }}$ and $V_{\text {data }}$ prior to the resistance drift of access transistors. As can be seen, the voltage margin is large enough to distinguish 
the resistance states between the data cell and reference cell, so there is no read bit errors in the first read operation shown in Fig.8-9.

For the second read operation after $15.1 \mathrm{~ns}$, the temporal transistors are turned on. When the data cell with logic ' 0 ' is read, the voltage difference between $V_{r e f}$ and $V_{p}$ becomes smaller than the pre-irradiation value, as shown in Fig.8. When logic ' 1 ' is read as illustrated in Fig.9, the negative voltage drift on data cell is larger than that on reference cell, then the initial value ' 1 ' is misread as ' 0 '. Fig. 10 shows the waveform of output $Q$ for this case.

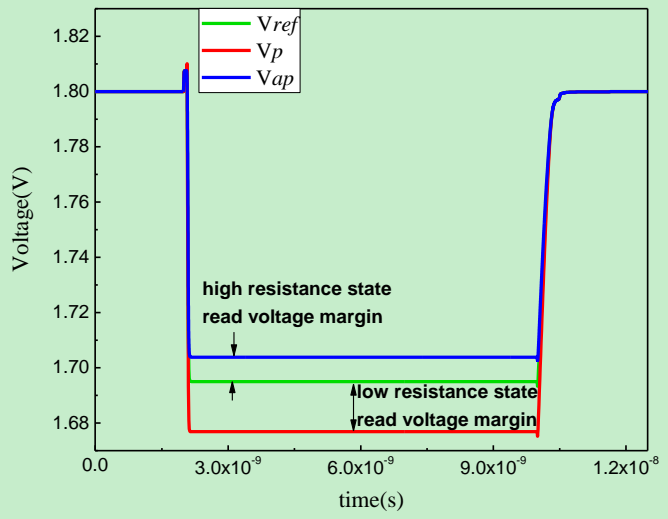

Fig.7.Voltage difference between $\mathrm{V}_{\text {ref }}$ and $\mathrm{V}_{\text {data }}$ in read operation without resistance drift

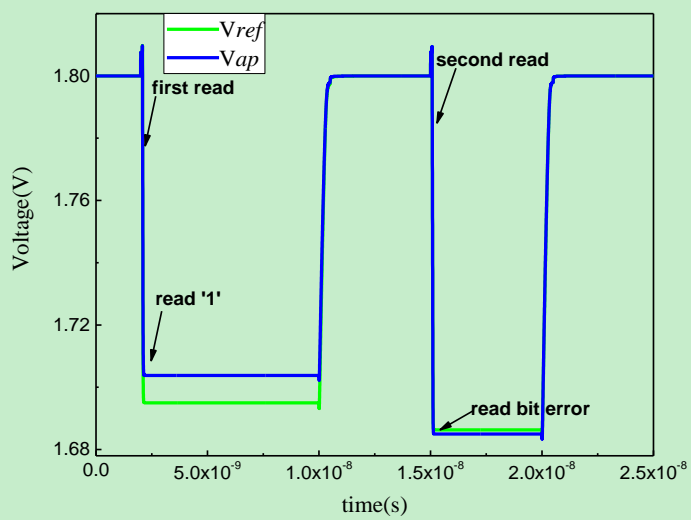

Fig.9. The variation of sensed voltage margin in read logic ' 1 ' operation before and after resistance drift

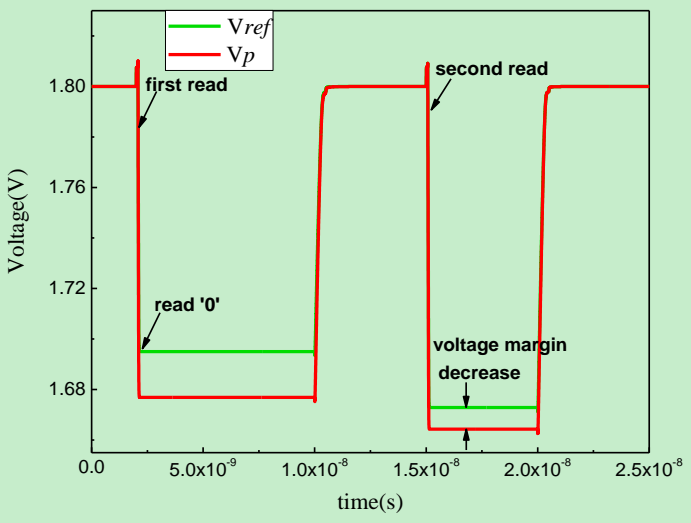

Fig.8. The variation of sensed voltage margin in read logic ' 0 ' operation before and after resistance drift

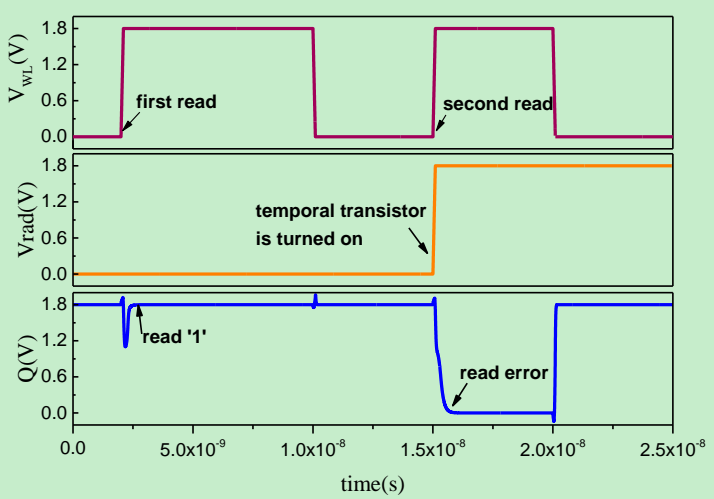

Fig.10.Waveform of read logic ' 1 ' operation before and after resistance drift

\section{Hardening Techniques for MRAM}

We have demonstrated that the read circuits may not be able to distinguish the low and high resistance states of a data cell in MRAM device, due to the radiation induced resistance drift of access transistors. To ensure the read operation of MRAM in a harsh radiation environment, hardening techniques may be applied by enlarging the resistance margins of $1 \mathrm{M} 1 \mathrm{~T}$.

\subsection{Optimization of MTJ performance}

In order to enlarge the resistance difference between high and low states of MTJ, a higher tunneling magneto-resistance (TMR) is preferred. The physics and dependency of TMR can be found in [28]. Process, material systems and device structures of MTJ can be optimized to achieve it ${ }^{[29,30]}$. 


\subsection{Optimization of access transistor performance}

As mentioned above, in order to diminish the effect of resistance drift on read margin, we can decrease the resistance of access transistor by enlarging W/L ratio. This way, the resistance ratio between MTJ and access transistor greatly increases, and thus the TID-induced resistance drift of access transistor can't lead to read errors. Literature ${ }^{[31]}$ shows that SRAM remained fully functional at high TID radiation levels by enlarging the width of transistors. However, wider transistors would increase storage cell size significantly and decrease the integration level.

Access transistor can also be hardened through layout techniques. For example, edgeless transistors along with guard-ring and/or guard-band ${ }^{[32]}$ have been proven to be effective in mitigating TID impacts in commercial CMOS technologies. The annular gate transistor can be also be used to replace the conventional access transistor.

Additionally, the STI oxide of access transistor may be processed to minimize the amount of charge traps, or we can use deep electron traps and recombination centers to produce as little net positive charges as possible ${ }^{[32,33]}$. Technologies such as ion implantation and layered films controlling the location of trapped charges also are effective in hardening STI region.

\section{Conclusion}

In this paper, TID-induced read bit errors in a $16 \mathrm{Mb}$ MRAM by Co60 have been observed in experiments and analyzed with simulations. Both 0-to-1 and 1-to-0 read bit errors have been observed when TID is accumulated over the threshold value. Resistance drift model of access transistor has been proposed to unveil the physical mechanisms of these upset errors in the MRAM device. The simulation results indicate that the TID-induced resistance drift of $1 \mathrm{M} 1 \mathrm{~T}$ has a significant impact on the read sensed voltage margin and is responsible for the read bit errors. This should be taken into consideration for the development of rad-hard MRAM products.

\section{Acknowledgements}

This work is partially supported by Chinese National Science Foundation under contract 61634008 and Youth Innovation Promotion Association CAS under contract 2014101. 


\section{Reference:}

[1] Bi jinshun, Liu Gang, Luo Jiajun, Han Zhengsheng. Numerical simulation of single-event-transient effects on ultra-thin-body fully-depleted silicon-on-insulator transistor based on $22 \mathrm{~nm}$ process node. Acta Phys. Sin. 2013;62(20):208501-8.

[2] Zhao Xing, Mei Bo, Bi Jinshun, Zheng Zhongshan, Gao Linchun, Zeng Chuanbin, Luo Jiajun, $\mathrm{Yu}$ Fang, Han Zhengsheng. Single event transients in a 0.18 um partially-depleted silicon-on-insulator complementary metal oxide semiconductor circuit. Acta Phys. Sin 2015; 64(13) :136102-8.

[3] T. R. Oldham, F. B. Mclean.Total ionizing dose effects in MOS oxides anddevices. IEEE Trans Nucl Sci 2003;50:483 - 99.

[4] Semiconductor Industry Association, "International Technology Road map for Semiconductors"http://itrs.net[2013].

[5] C. Augustine, A. Raychowdhury, D. Somasekhar, J. Tschanz, V. De, K. Roy. Design space exploration of typical STT MTJ stacks in memory arrays in the presence of variability and disturbances.IEEE Trans. Electron Devices2011;58(12): 4333-4343.

[6] I. Herrera-Alzu, M. Lopez-Vallejo. Design Techniques for Xilinx Virtex FPGA Configuration Memory Scrubbers. IEEE Trans. Nucl. Sci.2013;60(1):376-385.

[7] J. S. Shah, D. Nairn, M. Sachdev. A 32kb Macro with 8T Soft Error Robust SRAM cell in 65-nm CMOS. IEEE Trans. Nucl.Sci.2015; 62(3):1367-1374.

[8] H. Noguchi, K. Ikegami, K. Kushida, K. Abe, S. Itai, S. Takaya, N. Shimomura, J. Ito, A. Kawasumi, H. Hara, S. Fujita. 7.5A 3.3ns-access-time $71.2 \mathrm{uw} / \mathrm{MHz} 1 \mathrm{Mb}$ embedded STT-MRAM using physically eliminated read-disturb scheme and normally-off memory architecture. 2015IEEE Solid-State Circuits Conference, San Francisco,CA, Feb. 22-26, 2015 p1-3.

[9] W. S. Zhao, Y. Zhang, Y. Lakys, J. Klein.Embedded MRAM for high-speed computing. $19^{\text {th }}$ International Conference on VLSI and System-on-Chip, Hong Kong, Oct. 3-5, 2011,p37-42.

[10] B. Dieny, R. Sousa, S. Bandiera et al.. Extended scalability and functionalities of MRAM based on thermally assisted writing. 2011, IEDM,p1.3.1-1.3.4.

[11] Fanghui Ren, A. Jander, P. Dhagat, C. Nordam. Radiation tolerance of magnetic tunnel junctions with $\mathrm{MgO}$ tunnel barriers.IEEE Trans. Nucl. Sci.2012;59(6):3034-3038.

[12] D. Kobayashi,Y. Kakehashi,K. Hirose et al. Influence of heavy ion irradiation on perpendicular-anisotropy $\mathrm{CoFeB}-\mathrm{MgO}$ Magnetic Tunnel Junctions.IEEE Trans. Nucl. Sci.2014;61(4):1710-1716.

[13] H. Hughes, K. Bussmann, P. J. McMarr, S. F. Cheng, R. Shull, A. P. Chen, S. Schafer, T. Mewes, A. Ong, E. Chen, M. H. Mendenhall, R. A. Reed. Radiation studies of spin-transfer torque materials and devices. IEEE Trans. Nucl. Sci.2012;59(6):3027-3033.

[14] Jason Heidecker, G. Allen, D. Sheldon. Single event latchup(SEL) and total ionizing dose(TID) of a 1 Mbit magnetoresistive random access memory(MRAM). IEEE Radiation Effects Data Workshop 2010;p4-4.

[15] D. N. Nguyen, F. Irom. Radiation effects on MRAM. $9^{\text {th }}$ European Conference on Radiation and Its Effects on Components and Systems, RADECS 2007;p1-4. 
[16] Brady F T, Maimon J, Hurt M. A scaleable radiation hardened shallow trench isolation. IEEE Trans. Nucl. Sci.1999;46(6):1836-1840.

[17] Manghisoni M, Ratti L, Re V, Speziali V, Traversi G, Can-delori A. Comparison of ionizing radiation effects in $0.18 \mathrm{um}$ and $0.25 \mathrm{umCMOS}$ technologies for analog applications. IEEE Trans. Nucl. Sci..2003; 50(6):1827-1833.

[18] Re V, Manghisoni M, Ratti L, Speziali V, Traversi G. Total ionizing dose effects on the noise performance of a 0.13um CMOS technology.IEEETrans. Nucl. Sci. 2006;53(3):1599-1606.

[19] Liu L Z, Hu Z Y, Zhang Z X, Sao Hua, Ning B X, Bi D W, Chen Ming, Zhou S C. Total ionizing dose effect of 0.18um nMOSFETs.Acta Phys. Sin.2011;60(11):116103-5.

[20] Federico Faccio, Hugh J. Barnaby et al. total ionizing dose effects in shallow trench isolation oxides. Microelectron Reliab 2008;48(7)1000-1007.

[21] Behzad Razavi, 2001, Design of Analog CMOS Integrated Circuits(Published by McGraw-Hill): p17 - 26.

[22] Zhou Hang, Cui J W, Zheng Q W, Guo Qi, Ren D Y, Yu X F. Reliability of partially-depleted silicon-on-insulator $\mathrm{n}$-channel metal-oxide-semiconductor field-effect transistor under the ionizing radiation environment.Acta Phys. Sin.2015;64(8):086101-7.

[23]Lelis AJ, Oldham TR, Delancey WM. Response of interface traps during hightemperature anneals. IEEE Trans Nucl Sci 1991;38(6):1590 - 7.

[24] P. M. Figueiredo and J. C. Vital, Offset Reduction Techniques in High-Speed Analog-to-Digital Converters: Analysis,Design and Tradeoffs.Springer Science \& Business Media, 2009.

[25] Wang Zujun, Liu Changju, Ma Yan et al.. degradation of CMOS APS Image Sensors Induced by Ionizing Dose Radiation at different Dose Rates and Biased Conditions. IEEE Trans. Nucl. Sci.2015; 62(2):527-533.

[26] J. Kim, A. Chen, B. Behin-Aein, S. Kumar, J.P. Wang, and C.H. Kim. A Technology-Agnostic MTJ SPICE Model with User-Defined Dimensions for STT-MRAM Scalability Studies, Custom Integrated Circuits Conference (CICC), Sep. 2015.http://mtj.umn.edu/MTJ.html

[27] Jinsik Yun, J. Djigbenou, Dong S. Ha. HSPICE-Analog SPICE Simulation. http://www.mics.ece.vt.edu/ICDesign/Tutorials/Synopsys/hspice.html.

[28] J. Hayakawa, S. Ikeda, F. Matsukura, H. Takahashi, and H. Ohno, Dependence of giant tunnel magnetoresistance of sputtered $\mathrm{CoFeB} / \mathrm{MgO} / \mathrm{CoFeB}$ Magnetic tunnel junctions on $\mathrm{MgO}$ barrier thickness and annealing temperature. Jpn. J. Appl. Phys.,2005; 44: L587-L589.

[29] C. Sterwerf, M. Meinert et al., High TMR Ratio in $\mathrm{Co}_{2} \mathrm{FeSi}$ and $\mathrm{Fe}_{2} \mathrm{CoSi}$ Based Magnetic Tunnel Junctions. IEEE Trans. Magnetics.2013;49(7):4386-4389.

[30] Y. Wang, J. Qiu, R. Ji et al., High TMR ratio in perpendicular MTJs using fe-based Heusler alloy Fe2Cr1-xCoxSi. IEEE Magnetics Conference(INTERMAG).2015; p1-1.

[31] Xiaoyin Yao, Nathan Hindman, Lawrence T. Clark et al. The Impact of Total Ionizing Dose on Unhardened SRAM Cell Margins. IEEE Trans. Nucl. Sci.,2008;55(6):3280-3287.

[32] H. L. Hughes, J. M. Benedetto. Radiation Effects and Hardening of MOS Technology: Devices and Circuits. IEEE Trans. Nucl. Sci., 2003;50(3):500-521.

[33] H. Hatano, Radiation hardened high performance CMOS VLSI circuit designs. IEEE Proceedings G-Circuits, Devices and Systems.1992;139(3):287-294. 